\title{
Calidad de vida percibida por el personal de enfermería de las unidades de cuidados intensivos de una clínica privada de la ciudad de Santa Marta'
}

\author{
Yamile Puello Viloria², Nini Quintero Ramírez³, Carlos Canova \\ Barrios4 ${ }^{4}$ Yudis Camargo Mejía ${ }^{5}$, Laura Amaya Hernández6, \\ Yulieth Guzmán Galván7, Stephany Cervantes Polo
}

doi:10.11144/Javeriana.IE16-1.cvpp

Cómo citar: Puello Viloria Y, Quintero Ramírez N, Canova Barrios C, Camargo Mejía Y, Amaya Hernández L et al. Calidad de vida percibida por el personal de enfermería de las unidades de cuidados intensivos de una clínica privada de la ciudad de Santa Marta. Investig Enferm. Imagen Desarr. 2014;16(1): 87-99. doi:10.11144/Javeriana.IE16-1.cvpp

1. Artículo de investigación. Recibido: 24 de octubre de 2013. Revisado: 14 de enero de 2014. Aprobado: 10 de febrero de 2014.

2. Enfermera especialista en Adaptación y Rehabilitación Cardiopulmonar y Vascular. Docente catedrática médico-quirúrgica II y psiquiatría, Universidad del Magdalena, Santa Marta. Colombia. Correo electrónico: yampue2@hotmail.com.

3. Enfermera especialista en Gerencia y Auditoria en Salud. Docente catedrática, Universidad del Magdalena, Santa Marta, Colombia. Correo electrónico: niniquintero@hotmail.com.

4. Estudiante del programa de Enfermeria con énfasis en Cuidados Intensivos. Miembro del Semillero de Investigación adscrito al grupo Ciencias del Cuidado de Enfermería (GICCE) del programa de Enfermeria, Universidad del Magdalena, Santa Marta, Colombia. Correo electrónico: enfermeronice@hotmail.com.

5. Estudiante del programa de Enfermería (IX semestre), Universidad del Magdalena, Santa Marta, Colombia. Correo electrónico: yudis.05@hotmail.com.

6. Estudiante del programa de Enfermería (IX semestre), Universidad del Magdalena, Santa Marta, Colombia. Correo electrónico: laurysah_21@hotmail.com.

7. Estudiante del programa de Enfermería (IX semestre), Universidad del Magdalena, Santa Marta. Colombia. Correo electrónico: yulieth.guzman@hotmail.com.

8. Estudiante del programa de Enfermería (IX semestre), Universidad del Magdalena, Santa Marta, Colombia. Correo electrónico: tifany_1309@hotmail.com. 


\section{Resumen}

Introducción: la calidad de vida profesional (CVP) se relaciona con el equilibrio entre las demandas de trabajo y la capacidad percibida para llevarlas a cabo, a fin de garantizar el pleno desarrollo del ser humano en sus esferas laboral, familiar y personal. Objetivo: Describir la CVP por el personal de enfermería que labora en las unidades de cuidados intensivos adulto (UCI-A) y neonatal (UCI-N) de una institución de carácter privado de Santa Marta (Colombia), en 2013. Metodología: Estudio descriptivo de corte transversal, con enfoque cuantitativo. Se utilizó el cuestionario CVP-35 y una encuesta para los datos sociodemográficos del personal objeto de investigación. La muestra estuvo conformada por 34 trabajadores del área de enfermería de dos UCI: el $38,2 \%$ corresponde a profesionales de enfermeria, y el 61,7\%, al personal auxiliar. Resultados: Se identificaron diferencias respecto a la CVP percibida según el servicio en el que se labora y se situó al personal de la UCI-N dentro de rangos positivos de la escala de medición. Se encontró percepción de poco respaldo y compromiso por parte de quienes toman las decisiones relevantes hacia el personal inferior en las distintas jerarquías (apoyo directivo: 4,3/10). La dimensión mejor percibida fue la motivación intrínseca $(6,7 / 10)$, lo que demuestra conductas motivadas dirigidas a la satisfacción personal y la realización de las actividades del servicio por el placer de realizarlas, asî como una percepción disminuida de la carga laboral $(4,4 / 10)$, lo que evidencia calidad en los recursos de este campo aportados por la institución.

Palabras clave: calidad de vida, cuidados intensivos, personal de enfermería, motivación, carga de trabajo.

Palabras clave descriptor: calidad de vida, cuidados intensivos, personal de enfermería, motivación, carga de trabajo.

\section{Quality of life perceived by the nursing staff of the intensive care unit of a private clinic in the city of Santa Marta}

\section{Abstract}

Introduction: The Quality of Working Life (QWL) is related to the balance between work demands and the perceived ability to carry them out, to ensure the full development of human beings in their work, family and personal spheres. Objective: To describe the QWL by nurses working in the adult intensive care units (ICU-A) and neonatal (ICU-N) of a private institution of Santa Marta (Colombia) in 2013. Methodology: A descriptive cross-sectional study with a quantitative approach. The QWL-35 questionnaire and a survey for demographic data of the personnel under investigation were used. The sample consisted of 34 workers of nursing from two ICU: $38.2 \%$ were nurses, and $61.7 \%$, auxiliary personnel. Results: Differences regarding QWL were perceived according to the service in which personnel work and it placed the staff of the ICU-N within positive ranges in the measuring scale. It was found that there was perception of little support and commitment by relevant decision makers towards the lower staff in various hierarchies (managerial support: 4.3/10). The dimension perceived as the best was intrinsic motivation $(6,7 / 10)$, which demonstrates motivated behavior directed to personal satisfaction and fulfillment of service activities for the pleasure of carrying them out, and decreased perception of workload $(4,4 / 10)$, which demonstrates the quality of this field resources provided by the institution.

Keywords: quality of life, intensive care nurses, motivation, workload.

Key words plus: quality of life, intensive care, nursing staff, motivation, workload. 


\section{Qualidade de vida percebida pelo pessoal de enfermagem das unidades de tratamento intensivo de uma clínica privada da cidade de Santa Marta}

\section{Resumo}

Introdução: A qualidade de vida profissional (CVP) é relacionada com o equilíbrio entre as demandas de trabalho e a capacidade percebida para levá-las a cabo, a fim de garantir o pleno desenvolvimento do ser humano em suas esferas laboral, familiar e pessoal. Objetivo: Descrever a CVP pelo pessoal de enfermagem que trabalha nas unidades de tratamento intensivo adulto (UTI-A) e neonatal (UTI-N) de uma instituição de carácter privado de Santa Marta (Colômbia), em 2013. Metodologia: Estudo descritivo de corte transversal, com enfoque quantitativo. Utilizou-se o questionário CVP-35 e um inquérito para os dados sociodemográficos do pessoal objeto de pesquisa. A amostra esteve conformada por 34 trabalhadores da área de enfermagem de dois UCI: o 38,2\% corresponde a profissionais de enfermagem e o 61,7\% a pessoal auxiliar. Resultados: Foram identificadas diferenças no que diz respeito à CVP percebida de acordo ao serviço em que labora e foi situado o pessoal da UCI-N dentro de rangos positivos da escada de aferição. Encontrou-se percepção de pouco apoio e comprometimento dos tomadores de decisões relevantes para o pessoal menor nas várias hierarquias (apoio diretivo: 4,3/10). A dimensão melhor percebida foi a motivação intrínseca (6,7/10), o que mostra condutas motivadas encaminhadas à satisfação pessoal e a realização das atividades do serviço pelo prazer de realiza-las, tal como uma percepção diminuída da carga laboral $(4,4 / 10)$, o que evidencia qualidade nos recursos deste campo aportados pela instituição.

Palavras-chave: qualidade de vida, terapia intensiva, pessoal de enfermagem, motivação, carga de trabalho.

Palavras chave descriptor: qualidade de vida, terapia intensiva, recursos humanos de enfermagem, motivação, carga de trabalho. 


\section{Introducción}

La motivación laboral es el resultado de la relación entre el esfuerzo individual del trabajador, el rendimiento obtenido en el trabajo, las recompensas organizacionales al desempeño y los objetivos individuales de crecimiento personal/profesional (1). En la actualidad, los profesionales sanitarios laboran en un entorno cada vez más demandante, lo cual — sumado a la burocracia administrativa, que cada vez tiene más exigencias al profesional-, hace que pase a un segundo plano la llamada calidad de vida profesional (CVP), aumente la creciente responsabilidad clínica y administrativa y se minimice la autonomía en la toma de decisiones y capacidad resolutiva. El estrés, la insatisfacción con el tipo de trabajo, la carga laboral y el sueldo son factores condicionantes de la percepción del trabajador sobre su empleo y la CVP, en conjunto con la falta de oportunidades para el desarrollo profesional, expectativas insatisfechas e inadecuados incentivos por parte de la organización (2-5).

Cabeza (2) asevera que la CVP se relaciona con el equilibrio entre las demandas de trabajo y la capacidad percibida para llevarlas a cabo, a fin de garantizar el pleno desarrollo del ser humano en sus esferas laboral, familiar y personal; entre tanto, Fernández Araque y colaboradores (6), en una definición más amplia, definen la CVP como "el sentimiento de bienestar que se deriva del equilibrio que el individuo percibe entre las demandas o cargas de la profesión y los recursos psicológicos organizacionales y relacionales de que dispone para afrontar estas demandas" (7).

La satisfacción de los trabajadores de unidades sanitarias es uno de los objetivos de las organizaciones o instituciones a la que se hayan vinculados laboralmente, debido a que ellos constituyen la herramienta con la cual se asegura la prestación de servicios de calidad (8-10), debido a que los profesionales tienden a dar calidad al cliente/paciente/usuario, en la medida en que perciben calidad en los recursos organizacionales ofrecidos a él como trabajador (6).

Sin embargo, los profesionales de la salud presentan disposición a padecer el conocido síndrome de burnout o síndrome de desgaste profesional, relacionado directamente con características propias de la actividad laboral, incluido el contacto directo con el paciente, excesiva carga laboral, falta de motivación, inequidad o injusticia organizacional, imposibilidad de ascender en el trabajo, conflictos con los compañeros o usuarios, estrés, aburrimiento, pobres condiciones económicas, entre otras (11-13). El sindrome afecta de manera negativa la satisfacción del personal y la calidad de vida percibida por este, y ello influye en la calidad del trabajo ejecutado (14).

En las organizaciones se prioriza la calidad de vida del profesional, porque es un medio para garantizar la consecución de los objetivos organizacionales. Debido a esto, es necesario analizar y describir el estado percibido sobre la CVP para identificar qué aspectos mejorar, con la finalidad de asegurar el óptimo desarrollo de los trabajadores en las áreas profesional, familiar/ social y personal. Cabeza Peña (7) evalúa la CVP desde tres dimensiones: 
1. Carga de trabajo: percepción del trabajador respecto a las demandas de su trabajo, bajo los indicadores: cantidad de trabajo, prisas y agobios o presiones percibidas por la cantidad de trabajo o para mantener su calidad.

2. Motivación intrínseca: describe la motivación personal debido a factores internos que determinan la necesidad y, por tanto, la conducta motivada, dirigida a la satisfacción profesional. En esta dimensión se consideran: el tipo de trabajo que realiza, la motivación que experimenta, la exigencia de capacitación, el apoyo familiar y las ganas de ser creativo.

3. Apoyo directivo: se refiere al respaldo emocional que brindan los directivos a las personas que trabajan en la institución. Son considerados aspectos referentes a la posibilidad de expresión de sentimientos relacionados con el trabajo, autonomía o libertad de decisión, satisfacción con el sueldo, variedad del trabajo, reconocimiento del esfuerzo, oportunidad de promoción, apoyo de los jefes o compañeros y posibilidad de ser creativo.

El presente estudio describe la calidad de vida percibida por los trabajadores de enfermería en unidades de cuidado intensivo adulto y neonatal de una institución privada de la ciudad de Santa Marta (Colombia) en el segundo periodo de 2013. Sirve como una herramienta diagnóstica de la calidad de la atención brindada a los trabajadores en esta área bajo las dimensiones relacionadas con el apoyo directivo, la influencia de la carga de trabajo y la motivación intrínseca.

\section{Metodologia}

Tipo de estudio: estudio descriptivo de corte transversal, que permite describir la calidad de vida del personal de enfermería que labora en unidades de cuidados intensivos.

Muestra: estuvo conformada por el personal de enfermería que labora en las unidades de cuidados intensivos adulto (19 personas) y neonatal (15 personas) de una institución privada de la ciudad de Santa Marta. Un sujeto se negó a participar y esta muestra de 34 personas es representativa del $97 \%$ del personal total de enfermería en las unidades de cuidado intensivo de la clínica en cuestión.

Recolección de la información: se aplicó una encuesta divida en dos partes. La primera identifica el perfil sociodemográfico del participante y la segunda evalúa la calidad de vida percibida. Para esta segunda parte se implementó la versión española del QVP-35 (CVP-35) de Cabezas Peña (15), la cual tiene alta consistencia interna (alfa de Cronbach de 0,81). Este constructo mide la experiencia de bienestar derivada del equilibrio, que percibe el individuo frente a las demandas del trabajo y los recursos psicológicos organizacionales y relacionales que se disponen para afrontarlos, de manera que a lo largo de la vida se consiga un desarrollo óptimo de 
las esferas profesional, familiar y personal, enmarcado dentro de la CVP o laboral de los trabajadores $(16,17)$.

Este instrumento está dividido en tres subescalas que han alcanzado valores de confiabilidad de Cronbach mayores de 0,7. Este instrumento es autoadministrado, y se constituye por 35 items que se valoran en una escala tipo Likert, donde el sujeto evalúa en una escala de 10 adjetivos que van superpuestos al instrumento como guía las categorías nada ( 1 o 2), algo $(3,4$ y 5$)$, bastante $(6,7$ y 8$)$ y mucho (9 y 10).

Corrección de la prueba: está dividida en tres dimensiones y la pregunta referente a calidad de vida percibida, la cual se agrupa por fuera.

La primera dimensión (D1), compuesta por los ítems 2, 3, 4, 5, 10, $11,14,16,20,22,23$ y 28, evalúa el componente apoyo directivo, que hace referencia al apoyo emocional recibido por parte de los directivos.

La segunda dimensión (D2), compuesta por los items 1, 6, 7, 8, 17, 18, 19, 21, 24, 25 y 33, evalúa el componente carga de trabajo, el cual hace referencia a la percepción que el trabajador tiene de las demandas del puesto de trabajo.

La tercera dimensión (D3), compuesta por los ítems 9, 12, 13, 15, 26, 27, 29, 31, 32 y 35, evalúa la motivación intrínseca. Esta se orienta a establecer los factores de este tipo de motivación.

Por último, la CVP percibida es evaluada bajo el item 34.

Procesamiento de la información: se utilizaron medidas para el nivel descriptivo como frecuencias absolutas y desviación estándar (DE).

Resultados: se muestran los resultados calculados de la media aritmética y su respectiva DE.

Consideraciones éticas: se obtuvo el consentimiento informado de los participantes.

\section{Resultados}

Para la organización de los resultados se implementó el siguiente esquema:

1. CVP percibida de unidad de cuidados intensivo neonatal (UCI-N). General y discriminación por cargo.

2. CVP percibida profesional de unidad de cuidados intensivo adulto (UCI-A). General y discriminación por cargo.

3. CVP percibida. General y discriminación por servicio.

La D1 engloba el apoyo directivo; la D2, la percepción de la carga del trabajo; la D3, la motivación intrínseca, y la CVP, la calidad de vida profesional percibida.

\section{Unidad de cuidados intensivos neonatal}

La muestra de la UCI-N estuvo conformada por 15 trabajadores: una mues9) tra $100 \%$ femenina, de quienes el 53,3\% son profesionales de enfermería y el $46,7 \%$ es personal auxiliar de enfermería. La edad promedio es de 32,8 
años (DE: 6,4). La experiencia laboral promedio fue de 10,6 años (DE: 6) y la experiencia laboral en la UCI fue de 8,5 años (DE: 5,3). El estado civil estaba discriminado así: $46,6 \%$ casados, $40 \%$ solteros y $16,3 \%$ separados.

Los datos referentes a la CVP percibida son agrupados de manera general en la tabla 1. Se muestran los valores promediados y la DE.

TABLA 1. Calidad de vida percibida de los trabajadores de la UCI-N: general

\begin{tabular}{|l|c|c|}
\hline \multicolumn{1}{|c|}{ Dimensiones } & Promedio & DE \\
\hline D1 & 5,2 & 1,9 \\
\hline D2 & 3,7 & 1,0 \\
\hline D3 & 7,7 & 0,9 \\
\hline CVP & 6,0 & 2,6 \\
\hline
\end{tabular}

D1: apoyo directivo; D2: carga de trabajo; D3: motivación intrínseca; CVP: calidad de vida percibida.

FUENTE: elaboración propia.

De manera general, se perciben bajos puntajes en las dimensiones 1 y 2, mientras que la dimensión 3 y la CVP muestran puntajes aceptables o positivos de la escala de medición. Cabe destacar que es percibido poco apoyo por parte de los directivos, así como baja carga de trabajo, siendo este último un elemento positivo junto a la elevada motivación intrínseca y CVP.

Se realiza la discriminación de los datos promedio de la CVP percibida por cargo, subdividida la muestra entre profesionales de enfermería y auxiliares de enfermería (tabla 2).

TABla 2. Calidad de vida percibida en trabajadores de la UCI-N: discriminación por cargo

\begin{tabular}{|l|c|c|}
\hline \multicolumn{1}{|c|}{ Dimensiones } & $\begin{array}{c}\text { Profesionales } \\
\text { de enfermeria }\end{array}$ & Auxiliares de enfermeria \\
\hline D1 & 6,6 & 3,5 \\
\hline D2 & 3,4 & 4,0 \\
\hline D3 & 8,2 & 7,2 \\
\hline CVP & 6,8 & 5,1 \\
\hline
\end{tabular}

D1: apoyo directivo; D2: carga de trabajo; D3: motivación intrínseca; CVP: calidad de vida percibida.

FUENTE: elaboración propia.

Se encuentran niveles más altos en las dimensiones 1 y 3, así como en la calidad de vida profesional en los profesionales de enfermería y un mayor nivel en la dimensión 2, en el personal auxiliar, relacionado con el tipo de labores que realiza; sin embargo, solo se sitúa en la categoría "bastante" (rango positivo) a los trabajadores profesionales en las dimensiones 1 y CVP, y en general a los trabajadores en la D3 (motivación intrínseca). Se evidencia una percepción baja de la carga de trabajo. 


\section{Unidad de cuidados intensivos adulto}

La muestra de la UCI-A estuvo conformada por 19 trabajadores, de una muestra $100 \%$ femenina, constituida por un $26,3 \%$ de jefes de enfermería y un $73,6 \%$ de auxiliares. La edad promedio fue de 32,7 años (DE: 8,1), con una experiencia laboral promedio de 8,8 años (DE: 6,8) y una experiencia laboral en UCI de 3,9 años (DE: 3,1). El estado civil estaba discriminado así: $36,8 \%$ casados, $57,8 \%$ solteros y $5,26 \%$ viudos.

Los datos referentes a la calidad de vida profesional percibida son agrupados de manera general en la tabla 3 . Se muestran los valores promediados y la DE.

TABLA 3. Calidad de vida percibida de los trabajadores de la UCI-A: general

\begin{tabular}{|l|c|c|}
\hline \multicolumn{1}{|c|}{ Dimensiones } & Promedio & DE \\
\hline D1 & 4,3 & 1,1 \\
\hline D2 & 4,4 & 1,7 \\
\hline D3 & 6,7 & 1,1 \\
\hline CVP & 5,6 & 2,2 \\
\hline
\end{tabular}

D1: apoyo directivo; D2: carga de trabajo; D3: motivación intrínseca; CVP: calidad de vida percibida.

FUENTE: elaboración propia.

Se encuentran bajos niveles en la dimensión 1, un rango positivo en las categorías 2-3 y una calidad de vida intermedia para la muestra total.

Se realiza la discriminación de los datos promedio de la CVP percibida por cargo, subdividida la muestra entre profesionales de enfermería y auxiliares de enfermería (tabla 4).

TABla 4. Calidad de vida percibida en trabajadores de la UCI-A: discriminación por cargo

\begin{tabular}{|l|c|c|}
\hline \multicolumn{1}{|c|}{ Dimensiones } & $\begin{array}{c}\text { Profesionales } \\
\text { de enfermeria }\end{array}$ & Auxiliares de enfermeria \\
\hline D1 & 4,8 & 4,1 \\
\hline D2 & 5,0 & 4,1 \\
\hline D3 & 6,5 & 6,8 \\
\hline CVP & 5,8 & 5,5 \\
\hline
\end{tabular}

D1: apoyo directivo; D2: carga de trabajo; D3: motivación intrinseca; CVP: calidad de vida percibida.

FUENTE: elaboración propia.

Se encuentran niveles más altos en las dimensiones 1 y 2 , así como en la calidad de vida laboral en los profesionales de enfermería y un mayor nivel en la dimensión 3 en el personal auxiliar; sin embargo, solo se sitúa 94 en la categoría superior a los trabajadores en la dimensión 3. Se evidencia 
una percepción de carga laboral aumentada en el personal, con más énfasis en el personal profesional.

\section{Calidad de vida percibida: comparación entre UCI-N y UCI-A}

En esta parte se discriminaron los datos promedio de la calidad de vida profesional percibida por servicio, subdividida en UCI-N y UCI-A (tabla 5).

TABLA 5. Comparación entre la calidad de vida percibida de los trabajadores de UCI-N y UCI-N: discriminación por servicio (adulto y neonato)

\begin{tabular}{|l|c|c|c|}
\hline \multicolumn{1}{|c|}{ Dimensiones } & UCI-N & UCI-A & General \\
\hline D1 & 5,2 & 4,3 & 4,7 \\
\hline D2 & 3,7 & 4,4 & 4,0 \\
\hline D3 & 7,7 & 6,7 & 7,2 \\
\hline CVP & 6,0 & 5,6 & 5,8 \\
\hline
\end{tabular}

D1: apoyo directivo; D2: carga de trabajo; D3: motivación intrínseca; CVP: calidad de vida percibida.

FUENTE: elaboración propia.

Se encuentra mayor percepción de apoyo directivo (D1) y motivación intrínseca (D3) en la UCI-N y mayor carga de trabajo en la UCI-A. La calidad de vida percibida se sitúa en niveles positivos para la escala de medida en la UCI-N. De forma general, solo la dimensión motivación intrínseca es evaluada de manera positiva para los rangos de medición.

\section{Discusión}

El presente estudio se ha concentrado solo en una etapa diagnóstica inicial, pero es la base para estrategias institucionales enfocadas en mejorar la calidad de vida de sus trabajadores; además, se constituye en un corte longitudinal prospectivo para evaluar la eficacia de las estrategias implementadas, mejorar la calidad en el servicio suministrado por el personal, al ser este último el medio utilizado para cumplir los objetivos/metas de la institución.

Numerosos estudios hacen referencia al factor ingresos económicos como elemento protector de las personas frente a factores que puedan comprometer su homeostasis (18), y han sido hallados, al igual que en el presente estudio, más altos niveles de calidad de vida asociados a mayores ingresos económicos. Ello sitúa un punto de vista a la percepción positiva de la calidad de vida del personal profesional frente al auxiliar $(19,20)$.

Al mejorar la CVP se previene la aparición de enfermedades físicas y mentales, incluido el sindrome de burnout, pues se busca alcanzar el desarrollo humano y profesional pleno, mejorando la calidad de la atención suministrada por el personal a su usuarios (17). 
Al comparar los resultados de este estudio con similares, como el de Quezada (21), se encuentran similitudes en cuanto a la dimensión motivación intrínseca como la mejor evaluada y el apoyo directivo, el cual es percibido como bajo por el personal encuestado.

Las dimensiones apoyo directivo y carga laboral, así como la CVP, son puntuadas bajo la categoría "algo"; mientras la dimensión 3, motivación intrínseca, es puntuada bajo la categoría "bastante". Esto muestra el poco apoyo recibido por parte de sus superiores y las altas demandas de trabajo, que al ser contrastada con la definición de Cabezas (15), alude a un equilibrio entre las demandas de trabajo y la capacidad percibida para llevarlas a cabo como una evidencia de baja calidad de vida profesional; sin embargo, el personal mantiene la motivación al llevar a cabo sus obligaciones laborales.

El presente trabajo evalúa la calidad de vida percibida por los trabajadores usando el instrumento CVP-35, pero este instrumento no mide las expectativas de los trabajadores, lo cual es un elemento de suma importancia al momento de realizar las intervenciones necesarias para mejorar la CVP. Esta tiene unos niveles aceptables dentro del rango de medición y niveles similares han sido encontrados en personal de atención primaria en salud $(22,23)$; mientras los presentes son menores a los referenciados por otros estudios (21).

Estudios relacionados con la CVP percibida por un grupo de enfermeras y las consecuencias sobre su autocuidado refieren agotamiento o cansancio psíquico, inconformidades con el salario, así como actividades de entretenimiento, ocio, descanso y afecto minimizadas, debido a las altas demandas del trabajo (24).

En el presente estudio se muestran índices bajos de apoyo directivo y carga laboral, e índices positivos de motivación intrínseca. La CVP es evaluada bajo rangos negativos por la población general. Podría profundizarse y evaluar elementos propios de las actividades de apoyo directivo (autonomía, libertad de decisión, satisfacción con el sueldo, variedad del trabajo, reconocimiento al esfuerzo, oportunidades de promoción, posibilidad de ser creativo, apoyo por parte del personal directivo y los compañeros), carga laboral (cantidad de trabajo, presiones percibidas por la cantidad de trabajo o por mantener la calidad de este, duración de los turnos y horarios de trabajo) y motivación intrínseca (tipo de trabajo, motivación para laborar, exigencia de capacitación, apoyo familiar, objetivos de superación profesional y personal, ganas de ser creativo e innovar), para así detectar los elementos más influyentes en la percepción de calidad de vida profesional en la población encuestada, lo que constituye elementos clave para intervenir o fomentar, en busca de mejorar la calidad de vida profesional percibida por los trabajadores del área de enfermería en el área de cuidados intensivos, así como evaluar las consecuencias de estas dimensiones sobre las áreas profesional, psíquica, física, biológica, social, familiar y personal. 


\section{Conclusión}

Muestra constituida por 34 trabajadores del área de enfermería, 13 de ellos profesionales y 21 auxiliares, en su totalidad de sexo femenino, con edades promedio de 32,7 años, experiencia laboral de 10 años y 6 años de experiencia promedio dedicados al trabajo en la UCI.

En la UCI-N se encuentran niveles bajos en las dimensiones apoyo directivo (D1) y percepción de la carga de trabajo (D2), y alto en las dimensiones motivación intrínseca (D3) y CVP. En este servicio, el personal profesional de enfermería mostró puntuaciones superiores en las dimensiones 1, 3 y CVP, mientras que el personal auxiliar obtuvo cifras superiores en la dimensión carga de trabajo, relacionado con características propias de la actividad laboral que desempeñan.

En la UCI-A se encuentran niveles bajos en las dimensiones 1, 2 y CVP, y altos en la dimensión 3. En este servicio, el personal profesional obtuvo puntuaciones superiores en las dimensiones de apoyo directivo, carga de trabajo y CVP; mientras que el personal auxiliar demostró mayor motivación intrínseca.

Se detectan diferencias respecto a la CVP percibida según el servicio en el que se labora, y ello sitúa al personal de la UCI-N dentro de rangos positivos de la escala de medición. Se encuentra percepción de poco respaldo y compromiso por parte de quienes toman las decisiones relevantes hacia el personal inferior en los distintos niveles jerárquicos.

Debería asumirse un compromiso con las necesidades humanas viendo al personal no solo como un sistema productivo especializado, sino como eje principal de productividad empresarial, que busca conseguir mayor calidad y excelencia en el servicio suministrado, al darles a los trabajadores oportunidades de expresión y desarrollo.

La dimensión mejor percibida fue la de motivación intrínseca, lo que demuestra conductas motivadas dirigidas a la satisfacción personal y la realización de las actividades del servicio por el placer de realizarlas, así como una percepción disminuida de sobrecarga laboral, lo que evidencia calidad en los recursos de este campo aportados por la institución.

El personal mejor puntuado fue el de la UCI-N, evidenciado en menor percepción de carga laboral, mayor motivación intrínseca y calidad de vida profesional, situados en rangos positivos de la escala de medición usada. Respecto a la profesión, el personal profesional posee una mejor percepción de su calidad de vida en el trabajo frente al personal auxiliar.

\section{Referencias}

1. García S, González JA. Factores de motivación de los profesionales de la salud en atención primaria. FMC. 1995;2(1):1.

2. Vergara M. La renta médica en el sector público de salud. Revista Chilena de Salud Pública. 2001;5(2-3):102-10.

3. Goic A, Armas R. Descentralización en salud y educación: la experiencia chilena. Rev Med Chile. 2003;131(7):788-98. 
4. González A, Segovia I. La medicina general familiar en Chile. Cuad Med Soc (Chile). 1995;36 (no. extraordinario):29-33.

5. Katerndahl D, Parchman M, Wood R. Perceived complexity of care, perceived autonomy, and career satisfaction among primary care physicians. J Am Board Fam Med. 2009;22(1):24-33.

6. Fernández A, Jiménez S, Casado M. Calidad de vida de los profesionales de enfermería en atención primaria de Soria. Biblioteca Lascasas [internet]. 2007 [acceso 2014 mar 7];3(1). Disponible en: http:// www.index-f.com/lascasas/documentos/1c0213.php.

7. Cabeza C. Síndrome de desgaste profesional, estrés laboral y calidad de vida profesional. FMC. 1998;5(8):491-2.

8. Varo J. La calidad de la atención médica. Med Clin. 1995;104(14):538-40.

9. Sibbald B, Enzer I, Cooper C, Routh U, Sutherland V. GP job satisfaction in 1987, 1990, and 1998: lessons for the future? Fam Pract. 2000;17(5):364-71.

10. Newman K, Taylor U. The NHS plan: nurse satisfaction, commitment and retention strategies. Health Serv Manage Res. 2002;15(2):93105.

11. Cairo H. Burnout o estrés laboral: un síndrome cada vez más expandido. Mujeres de Empresa [internet]. 2002 [acceso 2013 sep 1]. Disponible en: http://www.mujeresdeempresa.com/relaciones_humanas/relaciones020402.shtm1.

12. Stortti M, Ayala EA, Span MS, Stortti MA. Etiología y prevención del síndrome de Burnout en los trabajadores de la salud. Revista de Posgrado de la VIa Cátedra de Medicina. 2006;153:18-21.

13. Perlman B, Hartman E. Burnout: summary and future research. Human Relations. 1982;35(4):283-385.

14. Meliá JL, Peiró JM. El cuestionario de satisfacción S10/12: estructura factorial, fiabilidad y validez. Rev Psicol Trab Org. 1989;4(11):17987.

15. Cabezas C. La calidad de vida de los profesionales. FMC. 2000;7 supl 7:53-68.

16. Aguilló E, Torres A. Calidad de vida laboral: hacia un enfoque integrador desde la psicología social. Psicotherma. 2002;14(4):828-36.

17. Grimaldo M. Calidad de vida profesional en estudiantes de ciencias de la salud. Rev Psicol. 2010;12:51-80.

18. Ahuvia A, Friedman D. Income, consumption, and subjective wellbeing: toward a composite macromarketing model. Journal of Macromarketing. 1998;18(2):153-68.

19. Tavallaii S, Einollahi B, Arizabadi Farahani M, Namdari M. Socioeconomic links to health-related quality of life, anxiety and depression in kidney transplant recipient. Iran J Kidney Dis. 2009;3(1):40-4.

20. Grimaldo M. Calidad de vida en los profesionales de la salud en la ciudad de Lima. Liberabit. 2011;17(2):173-85. 
21. Quezada F, Sanhueza A, Silva F. Diagnóstico de la calidad de vida laboral percibida por los trabajadores de cuatro servicios clínicos del complejo asistencial "Dr. Víctor Ríos Ruiz" de los ángeles (CAVRR). Horizontes Empresariales. 2010;9(1):55-68.

22. Clúa J, Aguilar C. La calidad de vida profesional y el orgullo de trabajar en la sanidad pública: resultados de una encuesta. Aten Primaria. 1998;22:308-13.

23. Sánchez R, Álvarez R, Lorenzo S. Calidad de vida profesional de los trabajadores de atención primaria del Área 10 de Madrid. Medifam. 2003;13(4):55-60.

24. Romero M, Meza L, Galindo S. Calidad de vida de las(os) profesionales de enfermería y sus consecuencias sobre el cuidado. Avances en Enfermería. 2008;26(2):59-70. 
